\title{
Ce qui marche, et ne marche pas en phytothérapie
}

Un opuscule d'une équipe spécialisée autour du Pr Paris avait pour titre "Ce qui marche, ce qui ne marche pas en phytothérapie ${ }^{1} »$. Édité en 2003, il proposait une revue de médications phytothérapiques qui ont montré des effets cliniques.

La distinction entre phytomédicament efficace et non efficace n'est pas aisée. Dans de nombreuses monographies sont indiquées des utilisations traditionnelles puisque les autorités n'admettent que peu les "indications" médicales " dures". Il $\mathrm{y}$ a en effet pour les différentes plantes médicinales davantage

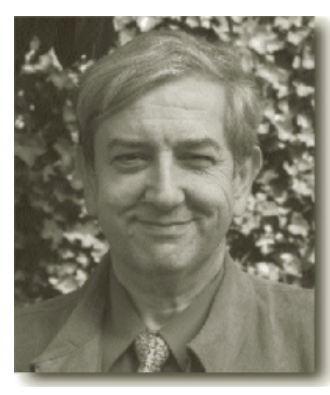
de travaux portant sur de la recherche pharmacologique pratiquée sur l'animal. Quelquefois il s'agit de travaux portant sur des pathologies provoquées par des substances toxiques (streptozotocine, alloxane, busulfan, adjuvant de Freund, carraghénine, etc.). Les expérimentations cliniques sont peu nombreuses mais ont augmenté en nombre et en qualité ces 20 dernières années. Souvent les recherches portent sur des drogues déjà connues, mais on a souvent aussi la bonne surprise de trouver des travaux sur des plantes moins utilisées. Bien sûr les plantes d'Orient n'arrivent que leñtement en France et il faudra apprendre à les connaître, quoique leurs "indications " correspondent souvent à une physiopathologie non occidentale.

Nous avons passé en revue un certain nombre de publications scientifiques représentant les recherches publiées pendant environ un quart d'année 2012 (Tableau 1), où lon peut voir la diversité des plantes étudiées, et les pays où se font les recherches.

Il est évident qu'il est difficile d'être un pharmacognoste " généraliste " ou un médecin pour lire toutes les communications. Notre rôle dans la revue est justement d'apporter au lecteur des condensés de ces dernières, ainsi que des " monographies " de drogues qui sont publiées le plus souvent en anglais pour le public scientifique. Au lecteur de se faire une idée en confrontant ses propres expériences et en trouvant des explications à des faits cliniques constatés ou en élargissant le domaine de l'application de ses "simples » préférés.

Il ne s'agit pas cependant d'appliquer toute nouvelle donnée scientifique portant sur l'animal. Ainsi, la découverte de certaines substances dans la gentiane qui pourraient avoir un effet antidépresseur a été surexploitée : et encore aujourd'hui aucune recherche clinique n'a été publiée à ce sujet.

La remise en ordre du DU de phytothérapie à la faculté Paris-XIII demande aux médecins et acteurs en phytothérapie de bien préciser quand il est possible de traiter une pathologie avec la seule phytoaromathérapie, et quand celle-ci ne peut être qu'un apport médicinal complémentaire au traitement conventionnel.

${ }^{1}$ Ce qui marche, ce qui ne marche pas en phytothérapie, éditions Josette, Lyon (épuisé). 


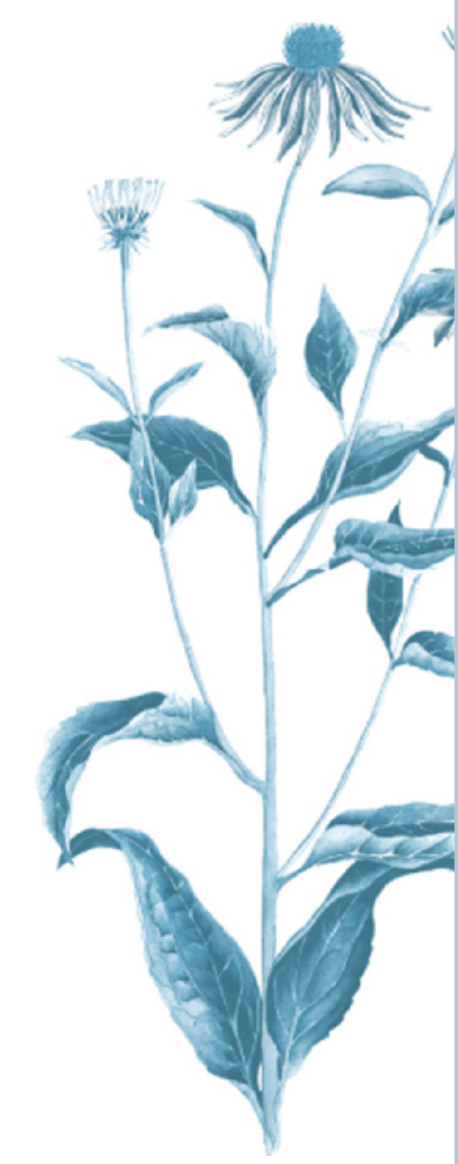




\begin{tabular}{|c|c|c|}
\hline Drogue & Recherche & Pays \\
\hline Lepidium sativum & Antidiarrhéique et antispasmodique & Arabie saoudite \\
\hline Withania somnifera, racines & Toxicité orale & Inde \\
\hline Schisandra sphenanthera & Pharmacocinétique de la cyclosporine A & Chine \\
\hline Miel de manuka et Portobello & Antimicrobien & Écosse \\
\hline Gracilaria verrucosa & Accumulation lipidique & Corée \\
\hline Aloe vera & Pathologie des tendons & Brésil \\
\hline Cissus quadrangularis & Évaluation & États-Unis \\
\hline Silibinine & Plaie cutanée & Iran \\
\hline Alisma orientale & Allergie & Corée \\
\hline Polygonatum falcatum, kaempferol & Adipogenèse & Corée \\
\hline Ulva, ulvane & Cytotoxicité & Portugal \\
\hline Asiaticoside & Antipyrétique et anti-inflammatoire & Chine \\
\hline Phyllanthus emblica & Prokinétique, laxatif et spasmodique & Pakistan \\
\hline Cimicifuga spp. & Ménopause & Australie \\
\hline Tabebuia avellanedae & Antiulcéreux & Brésil \\
\hline $\begin{array}{l}\text { Curcuma xanthorrhiza Roxb., } \\
\text { Xanthorrhizol }\end{array}$ & Candida albicans & Malaisie \\
\hline $\begin{array}{l}\text { Vanillylamide de l'acide pélargonique, } \\
\text { capsaïcine }\end{array}$ & Comparaison, récepteurs vanilloïdes & RFA \\
\hline Ginkgo biloba & Maladie d'Alzheimer & France \\
\hline Ginkgo biloba & Fonction cognitive & États-Unis \\
\hline$E g b>61$ & $\begin{array}{l}\text { Ostéoblastogenèse } \\
\text { Athérosclérose }\end{array}$ & Inde \\
\hline Schizonepeta tenuifolia & Dermatite atopique & Corée \\
\hline Beta vulgaris, en oral & Barrière cutanée & Japon \\
\hline Crocus sativus, crocine & Résistance à l'insuline & Iran \\
\hline Aronia melanocarpa & $\begin{array}{l}\text { Érythrocytes en cas } \\
\text { d'hypercholestérolémie }\end{array}$ & Pologne \\
\hline Tamarindus indica & Diabète & Inde \\
\hline Cecropia pachystachya & $\begin{array}{l}\text { Anti-inflammatoire, antinociceptif } \\
\text { et cytotoxique }\end{array}$ & Brésil \\
\hline Sanguisorba, racine & Neurotoxicité & Corée \\
\hline Cissampelos sympodialis & Allergie & Brésil \\
\hline Pistacia chinensis & Antioxydant & Corée \\
\hline Agaricus blazei, extrait & Anti-inflammatoire antiallergique & Corée \\
\hline Actinidia callosa var. ephippioides. & Anti-inflammatoire, antiproliférative & Taiwan \\
\hline Cimicifuga foetida & Virus syncytial & Taiwan \\
\hline Viola yedoensis & Affection pulmonaire & Chine \\
\hline Artemisia princeps var. orientalis & Mémoire & Corée \\
\hline Rubus coreanus & Oxydation du LDL & Corée \\
\hline Ferula hermonis & Antifongique & Espagne \\
\hline Gymnema sylvestre & Intolérance au glucose & Royaume-Uni \\
\hline $\begin{array}{l}\text { Cinnamomum philippinense, } \\
\text { Cinnamophiline }\end{array}$ & Dégradation des chondrocytes & Taiwan \\
\hline $\begin{array}{l}\text { Hypericum origanifolium } \\
\text { Etc. }\end{array}$ & Effet sur le SNC & Turquie \\
\hline
\end{tabular}

P. Goetz 